\title{
Down-regulation of 14q32-encoded miRNAs and tumor suppressor role for miR-654-3p in papillary thyroid cancer
}

\author{
Murilo Vieira Geraldo ${ }^{1,3}$, Helder Imoto Nakaya², Edna Teruko Kimura ${ }^{1}$ \\ ${ }^{1}$ Department of Cell and Developmental Biology, Institute of Biomedical Sciences, University of Sao Paulo, Sao Paulo, Brazil \\ ${ }^{2}$ Department of Clinical Analyses and Toxicology, School of Pharmaceutical Sciences, University of Sao Paulo, Sao Paulo, Brazil \\ ${ }^{3}$ Department of Structural and Functional Biology, Institute of Biology, State University of Campinas, Campinas, Brazil \\ Correspondence to: Murilo Vieira Geraldo, email: murilovg@unicamp.br \\ Edna Teruko Kimura, email: etkimura@usp.br
}

Keywords: microRNA, papillary thyroid carcinoma, 14q32 region, microTranscriptome, metastasis

Received: September 18, 2016

Accepted: November 26, 2016

Published: December 24, 2016

\section{ABSTRACT}

Papillary thyroid carcinoma (PTC) is the most prevalent malignant neoplasia of the thyroid gland. A fraction of PTC cases show loss of differentiation and aggressive behavior, with radioiodine therapy resistance and metastasis. Although microRNAs (miRNAs) emerged as promising molecular markers for PTC, their role in the loss of differentiation observed during PTC progression remains to be fully understood. We performed the large-scale analysis of miRNA expression during PTC progression in BRAFT1799A-transgenic animals ( $\mathrm{Tg}$-Braf) and thyroid cancer cell lines and identified the marked downregulation of several miRNAs from the region 14q32. Data from The Cancer Genome Atlas (TCGA) confirmed the global downregulation of miRNAs from the 14q32 region in human PTC. The regulatory network potentially suppressed by these miRNAs suggests that key cancer-related biological processes such as cell proliferation, adhesion, migration and angiogenesis. Among the downregulated miRNAs, we observed that miR-654-3p levels decrease with long-term PTC progression in Tg-Braf mice and inversely correlate with EMT. The in vitro restoration of $m i R-$ 654-3p decreased cell proliferation and migration and induced reprogramming of metastasis-related genes, suggesting a tumor suppressor role for this miRNA. In conclusion, we show global downregulation of 14q32-encoded miRNAs in an in vivo model of PTC progression. The potential circuitry in which these miRNAs are involved suggests that these miRNAs could play a key role in the pathophysiology of PTC and therefore be relevant for the development of new therapeutic strategies.

\section{INTRODUCTION}

In the last decade, an increasing number of thyroid cancer cases has been observed worldwide. More than 64,300 new cases of thyroid cancer are estimated to be diagnosed in 2016, leading to 1,980 deaths [1] in USA. The most prevalent subtype is papillary thyroid carcinoma (PTC), accounting for $80 \%$ of all cases. Although PTCs typically confer good prognoses, about $5 \%$ of PTC cases present as aggressive tumors, with radioiodine therapy resistance, lymph node metastatic dissemination and poor prognosis [2]. The most prevalent genetic alterations in thyroid tumors are BRAFT1799A mutation and RET/PTC chromosomal rearrangements, which have been extensively explored as prognostic markers and therapeutic targets [3-5].

miRNAs are small RNA molecules involved in post-transcriptional regulation of gene expression, mainly through imperfect base pairing with the $3^{\prime}$ untranslated region (UTR) of target messenger RNA (mRNA)[6]. Several studies have implicated differential expression of miRNAs as a promising molecular marker for aggressive and recurrent thyroid cancer [7-11]. Due to a short length and imperfect base-pairing, a single miRNA is predicted to regulate hundreds of target mRNAs. Conversely, several miRNAs can cooperate to regulate a single target. Thus, the contribution of multiple deregulated miRNAs to posttranscriptional regulation in thyroid cancer, particularly regarding aggressive PTC, remains poorly understood. 
In this study, we used Tg-Braf mice to assess the micro-transcriptome during PTC progression and identified downregulation of several miRNAs from the $14 q 32$ genomic region. This region spans $\sim 850 \mathrm{~kb}$ and harbors distinct imprinted genes (DLK1, RTL1, MEG3, $M E G 8$ and DIO3), C/D small nucleolar RNAs (SNORDs) and more than 50 miRNA genes, many described as tumor suppressors in different types of cancer $[12,13]$. The observation that a subset of Temple Syndrome (TS) patients, in which the $14 \mathrm{q} 32$ region is partially or completely deleted, present with increased risk of developing differentiated thyroid cancer suggests that some of the genes located in this region may modulate biological processes crucial for thyroid follicular cell transformation $[14,15]$. Our in silico analysis highlights miRNAs from the 14q32 locus as candidate targets in PTC. Furthermore, we show tumor suppressor properties for $m i R-654-3 p$ in vitro, which could open perspectives for new therapeutic strategies for PTC.

\section{RESULTS}

\section{Global downregulation of miRNAs from the $14 q 32$ genomic locus in a transgenic mouse model of PTC and human PTC samples}

To identify differentially expressed miRNAs during PTC loss of differentiation, we used a transgenic mouse model of PTC [16] that harbors a thyroid-targeted BRAFT1799A mutation (Figure 1a). While thyroid glands extracted from 5-weeks old mice exhibit classic well differentiated PTC, thyroid glands from older mice show foci of locally invasive poorly differentiated thyroid carcinoma [17]. Large scale analysis revealed that several miRNAs from the $14 \mathrm{q} 32$ genomic region are down-regulated during PTC progression (Figure 1b). Importantly, the miRNAs $m i R-495-3 p$, miR-654$3 p, m i R-376 a-3 p$ and $m i R-487 b-3 p$ exhibited marked downregulation after 5 weeks in contrast to a slight reduction of expression observed for most miRNA genes from this region. Downregulation of miRNAs from the $14 \mathrm{q} 32$ locus was also observed in thyroid cancer cell lines, prominently in BRAFT1799A-mutated BCPAP and KTC-2 cells, as compared to the normal thyroid cell line N-Thy-ORI (Figure 1b). As shown in Figure 1c, the locus 14q32 comprises 53 miRNA genes divided in two clusters, designated here as "miRNA cluster 1" (the larger cluster) and "miRNA cluster 2" (the smaller cluster). This region also comprises several copies of C/D small nucleolar RNAs (SNORDs) 112, 113 and 114, and the imprinted genes DLK1, RTL1, MEG3, MEG8 and DIO3. The TCGA portal (https://cancergenome.nih.gov) reveals a single patient with homodeletion of the entire $14 q 32$ region without any additional information regarding heterozygosity in thyroid cancer. The analysis of randomly-chosen miRNAs from clusters 1 and 2 (miR-654-3p, mir-369-3p, miR-495, $m i R-370-5 p$, $m i R-127-5 p$ and $m i R-376 c-3 p$ ) in tumor cell lines confirmed their downregulation (Figure 1d). Importantly, the reduced expression of miRNAs from the $14 \mathrm{q} 32$ locus could not be due to terminal deletion of the 3 ' end of chromosome 14, as the thyroid cancer cell lines expressed increased levels of $m i R-203 a$, situated $\sim 3 \mathrm{Mb}$ downstream of the $14 \mathrm{q} 32$ locus.

We then analyzed whether downregulation of genes located at the $14 \mathrm{q} 32$ genomic region is observed in human PTC samples. We downloaded mRNA and miRNA expression datasets from 57 PTC samples and matched non-tumor thyroid tissue from The Cancer Genome Atlas (TCGA) project, and observed the downregulation of most miRNAs from the 14q32 genomic region (Figure 2a). Importantly, this reduction of expression is restricted to genes situated between $D L K 1$ and DIO3. As shown in Figure $2 \mathrm{~b}$, we observed an orchestrated expression pattern for these miRNAs across samples, suggesting either the existence of a polycystronic primary miRNA transcript or regulation of the miRNA cluster by a common mechanism. The analysis of miRNA expression of the whole TCGA dataset, comprising 437 PTC samples and 59 normal thyroid tissue samples, also showed the global miRNAs downregulation located at the $14 \mathrm{q} 32$ region (Figure $2 \mathrm{c}$ ).

\section{Post-transcriptional regulatory network of miRNAs from the $14 \mathrm{q} 32$ region}

To understand the role of the miRNAs from the DLK1-DIO3 region in PTC pathogenesis, we analyzed the post-transcriptional regulatory network potentially modulated by these miRNAs in silico. As shown in Figure $3 \mathrm{a}$, were selected 28 differentially expressed miRNAs from the $14 \mathrm{q} 32$ region that give rise to 32 mature miRNA species. miRNAs with a low or absent number of reads were not considered for analysis. We used the miRWalk webtool to search for predicted targets of each of the 32 mature miRNAs in the 14q32 region. To increase the stringency of target prediction, a minimum of seven algorithms were considered as a cutoff for each miRNA:mRNA interaction. Four mature miRNAs were excluded due to the absence of targets predicted by seven or more algorithms. A total of 7,886 genes targeted by 28 miRNAs were included for Gene Set Enrichment Analysis (GSEA). As the miRNAs from 14q32 are downregulated in PTC, we compared the list of potential targets with RNA-seq V2 data from TCGA and searched for those presenting upregulation ( $>1.25$-fold) in PTC samples. The resulting list of 1,193 predicted targets was subsequently submitted to the DAVID webtool to identify enriched biological processes and signaling pathways. Sixty-seven GO Terms were enriched among the target genes ( $\mathrm{P}$ value $<0.001$; Supplementary Table 2). The most significantlyenriched biological processes were related to cell adhesion and motility, cell proliferation and vascular development (Figure 3b). Additionally, by comparing the lists of 
miRNA targets, we identified a significant enrichment for 15 out of the 28 miRNAs, suggesting that most of the 14q32-encoded miRNAs may cooperate to regulate the same biological processes (Figure 3c).

\section{Tumor suppressor properties of miR-654-3p in thyroid cell lines}

Among the differentially expressed miRNAs from the $14 \mathrm{q} 32$ region, we observed that miR-654-3p levels decrease with long-term PTC progression in Tg-Braf mice and inversely correlate with the expression of the EMT markers Zeb1, Zeb2, Snai1 and Snai2 (Figure 4a). The restoration of $m i R-654-3 p$ expression using commercial mimetic miRNA markedly decreased cell proliferation and migration, and increased apoptosis in normal and tumor thyroid cell lines (Figure $4 \mathrm{~b}$ and $4 \mathrm{c}$ ). Importantly, a cell migration assay was performed $24 \mathrm{~h}$ after miR-654-3p transfection, ensuring that the observed effect was due to migration impairment rather than decreased proliferation.

To evaluate the participation of $m i R-654-3 p$ in PTC progression, we analyzed the impact of ectopic expression a

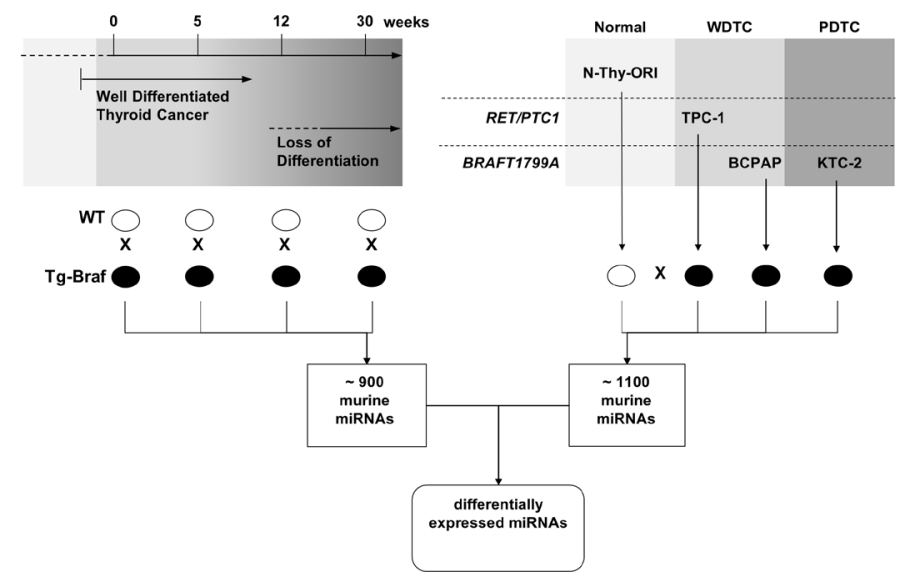

b
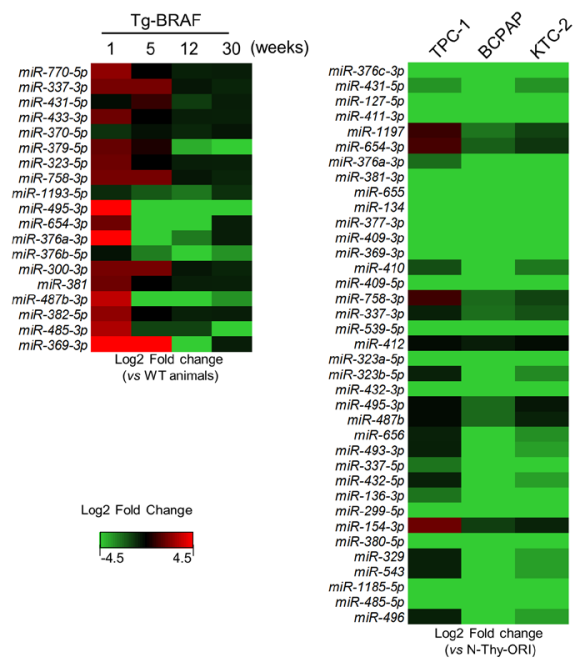

d
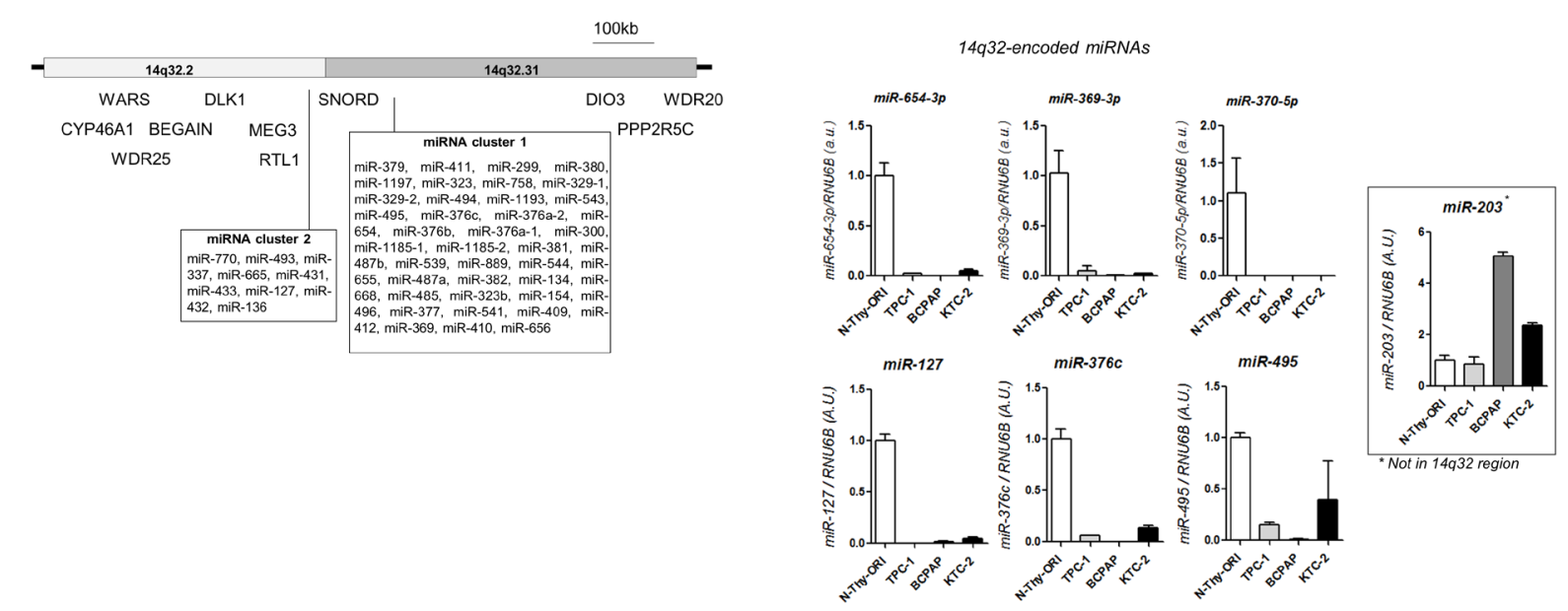

Figure 1: MiRNAs from 14q32 region are down regulated in thyroid cancer cell lines and in murine model of PTC. a. Large scale analysis of PTC progression In vivo and in vitro. Panel of thyroid cell lines including normal (N-Thy-ORI), PTC cell line bearing RET/PTCl oncogene (TPC-1), and PTC and ATC cell lines bearing BRAFT1799A oncogene (BCPAP and KTC-2, respectively). Expression of miRNAs from transgenic animals in each time point was compared with respective wild-type sample. Expression of miRNAs for each thyroid cancer cell line was compared with the normal cell line N-Thy-ORI. b. Downregulation of 14q32-encoded miRNAs in Tg-Braf mouse model and thyroid cancer cell lines. Heatmap represents the fold change between each thyroid cancer cell line and normal cells (N-Thy-ORI) and between Tg-Braf and Wild-type animals in each time point. c. Schematic representation of DLK1-DIO3 region and miRNA clusters in 14q32 chromosome locus. The distances of 5' and 3' flanking genes CYP46A1, WARS, WRD25, BEGAIN, PPP2R5C and WRD20 are not in scale. d. Validation of miRNAs from $14 \mathrm{q} 32$ region by independent qPCR assay. Increased expression of $m i R-203$, which is located downstream of DLK1-DIO3, shows absence of terminal deletion of chromosome 14. Small RNA U6B (RNU6B) gene was used as endogenous control. 
of $m i R-654-3 p$ on the expression of several genes involved in tumor establishment and progression using a Taqman $\mathbb{}$ Human Tumor Metastasis qPCR Array. The miR-654$3 p$-transfected cells showed increased expression levels of the epithelial cell markers $C D H 1$ and $C T N N A 1$ and decreased levels of $S N A I 2$, with no significant changes in ZEB1, ZEB2, SNAI1 and VIM levels (Figure 4d). No morphological changes were observed through light microscopy after transfection of $m i R-654-3 p$ (data not shown). Known tumor suppressor genes were upregulated after transfection of mimetic miR-654-3p, including CASP8, PTEN, DAPK1, RB1 and the cell cycle regulator $C D K N 2 A$. Moreover, key genes involved in cell adhesion, migration and ECM remodeling were downregulated after transfection of mimetic $m i R-654-3 p$, such as $L A M B 1$ and MMP9. Also, decreased levels of HRAS, FGF2,

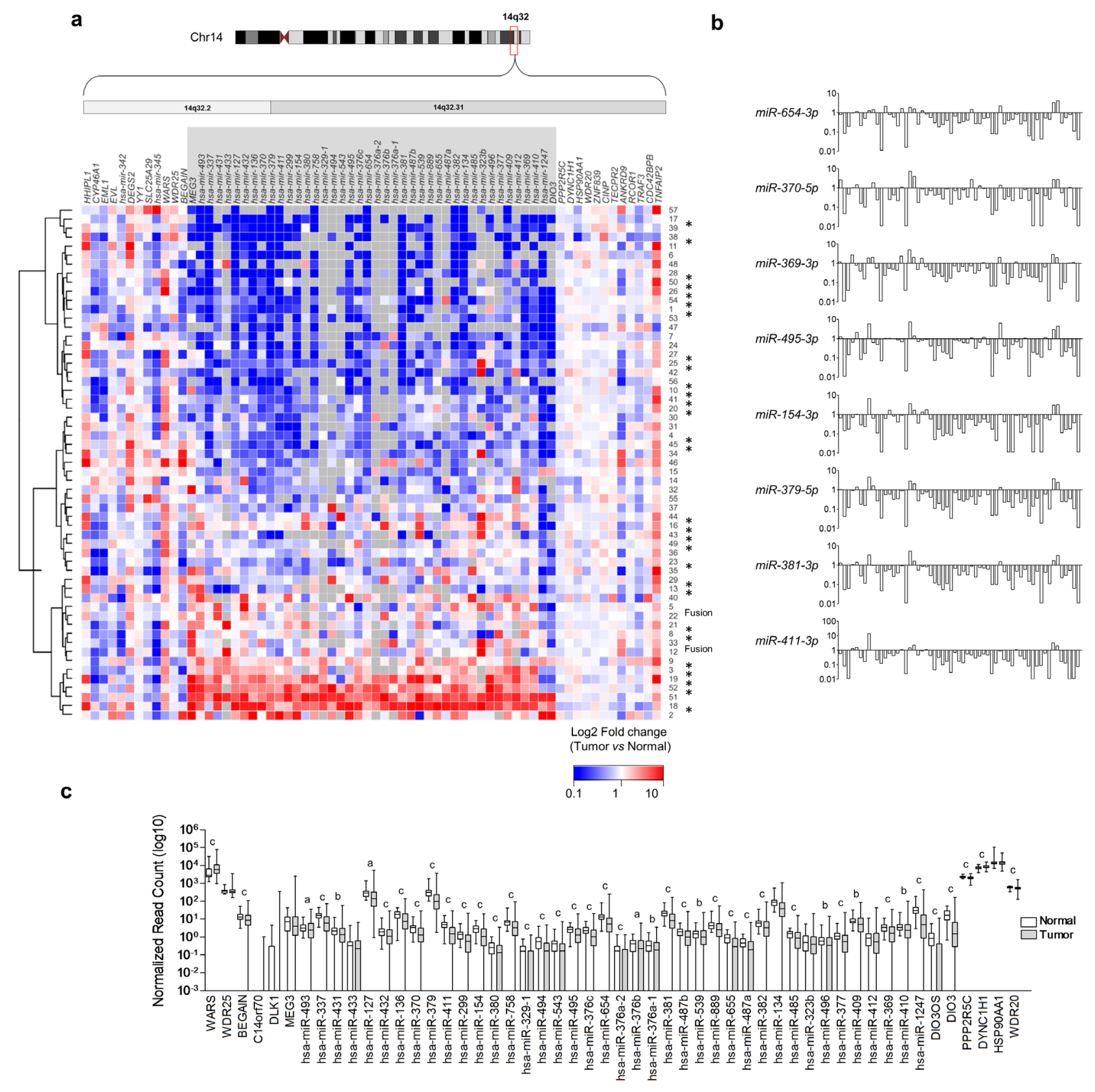

Figure 2: Global downregulation of miRNAs from 14q32-encoded genes in PTC. a. Gene expression data from 57 paired samples downloaded from TCGA website was used to construct the heatmap. Grey boxes represent null values for sequence read either in Normal or Tumor sample. (*) BRAFT1799A mutation; (Fusion) BRAF gene fusion. b. Analysis of 57 paired PTC and normal thyroid tissue reveals concordant miRNA expression pattern across samples. Samples with null values either for PTC or normal tissue were replaced by the lowest value for the given gene. c. Average expression of miRNAs from DLK1-DIO3 region and flanking genes in 437 PTC samples compared with 59 normal thyroid samples. (a) p-value $<0.05$; (b) p-value $<0.01$; (c) p-value $<0.001$. 
FGFR4 and $I L 1 B$, as well as increased $N F 1$, components of MAPK signal transduction, were observed after transfection of $m i R-654-3 p$. Importantly, restoration of $m i R-654-3 p$ upregulated the expression of the metastasis- suppressor genes BRMS1, MTSS1, KISS1 and NME1, and downregulated the expression of several genes related to tumor progression and metastasis, including MTA1, MTA2, SERPINE1, SSTR2 and CD44. Altogether, these data a
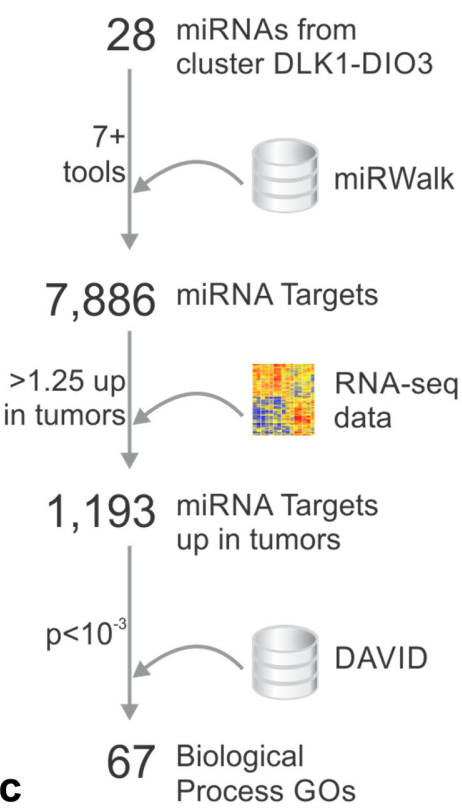

b

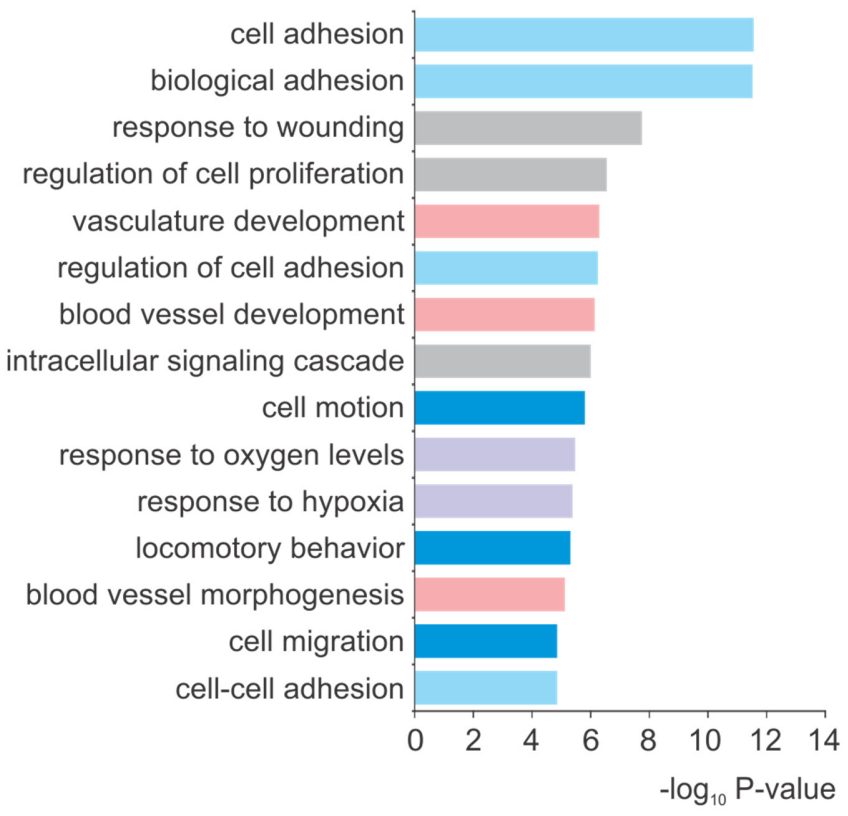

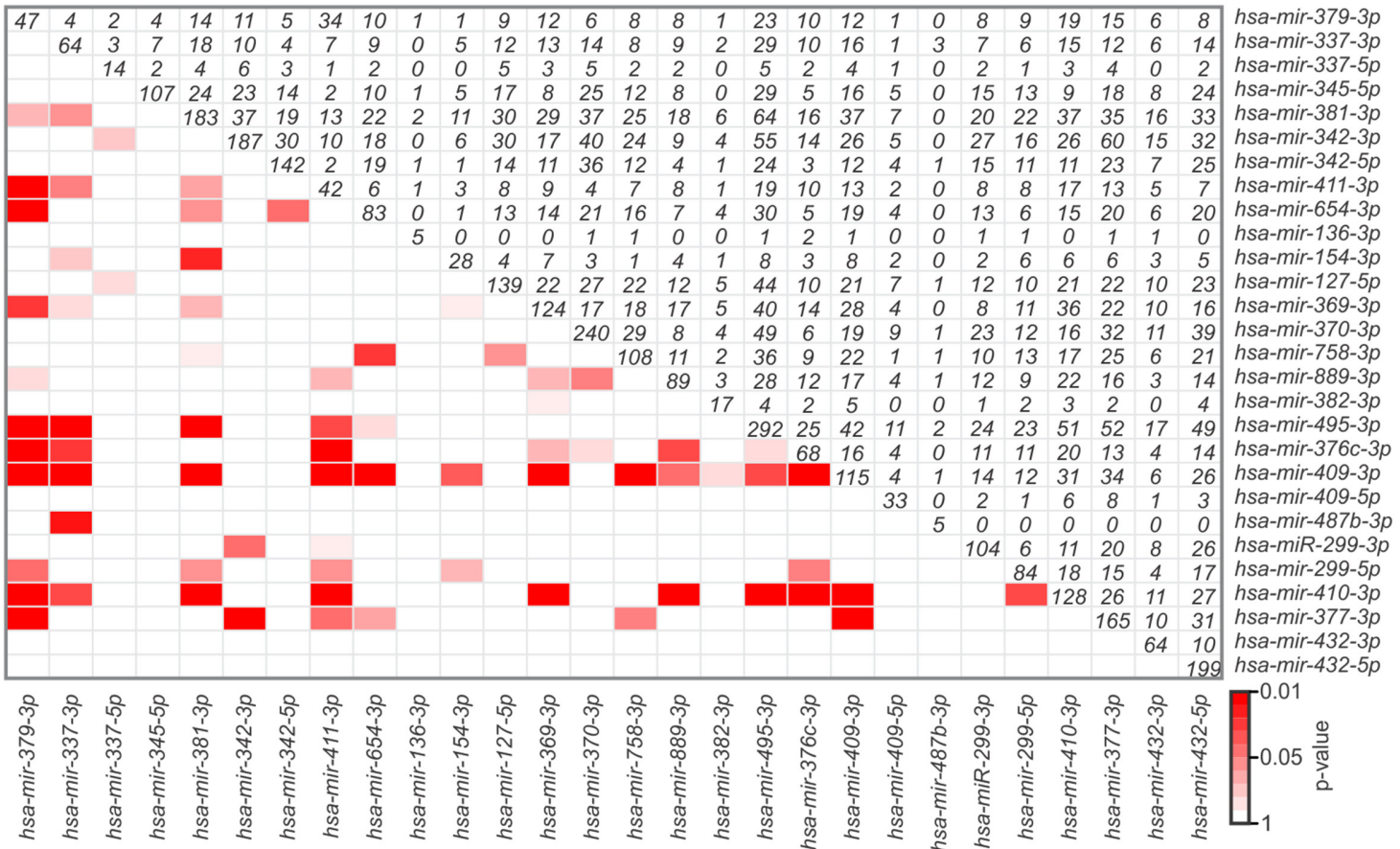

Figure 3: miRNAs from $14 q 32$ region potentially modulate cancer-related processes. a. The prediction of miRNA targets by at least 7 algorithms was performed using miRWalk webtool. RNA-Seq data was searched for upregulated genes (>1.25 fold) and the resulting list was submited to DAVID webtool. b. GO terms enriched among the list of potential targets of 14q32-encoded miRNAs. c. miRNAs from DLK1-DIO3 region target common genes. Lower triangular matrix shows the p-value of either Fisher's Exact Test of Chi-square (if sample size is too large for the Fisher test). Upper triangular matrix shows the number of targets shared by both miRNAs. Diagonal numbers are the total number of targets for a given microRNA. 
suggest that restoration of $m i R-654-3 p$ could contribute to a more differentiated, less aggressive phenotype in PTC.

\section{DISCUSSION}

Although the abnormal expression of miRNAs has been widely described in well-differentiated thyroid cancer, the role of these molecules in PTC progression remains unclear. In silico and experimental analyses suggest that abnormal miRNA expression may participate in key biological processes during cancer invasion and dissemination, and can contribute to aggressive behavior of PTC [10, 18-20]. Using an in vivo mouse model of PTC, we identified marked a

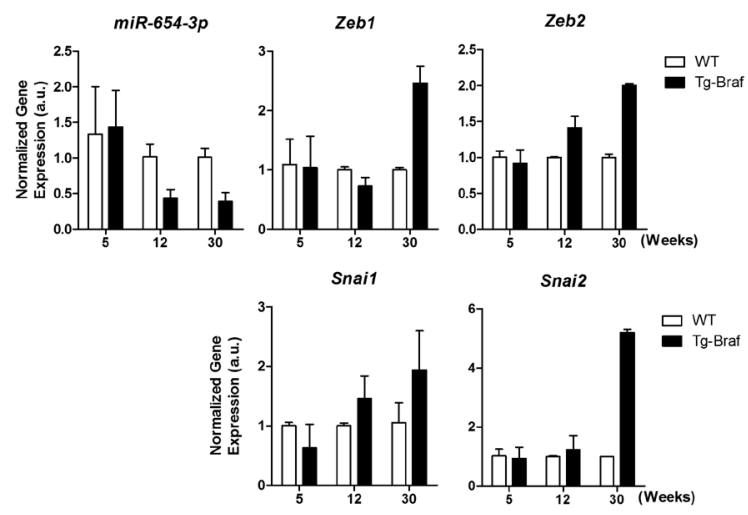

b
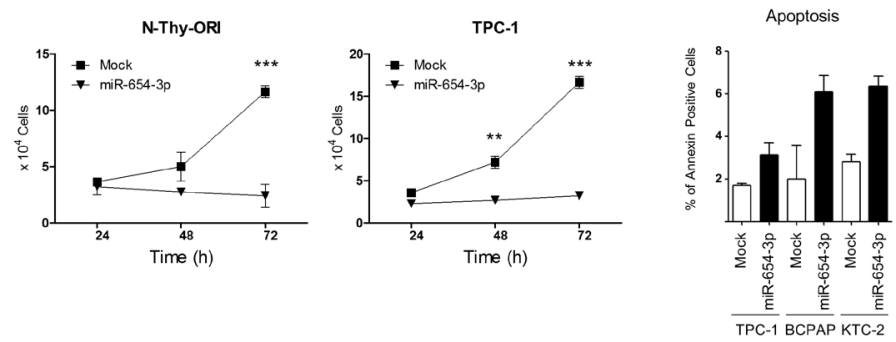

C

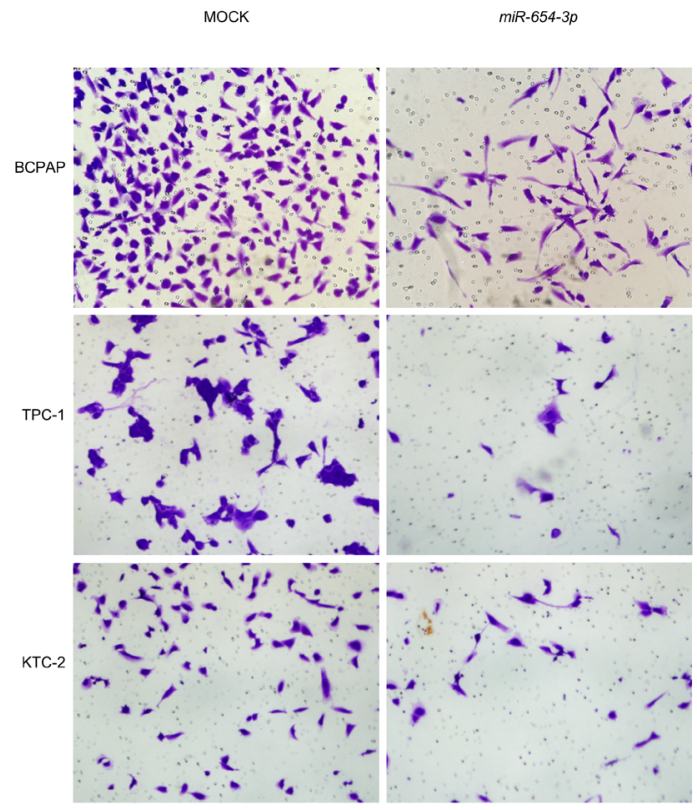

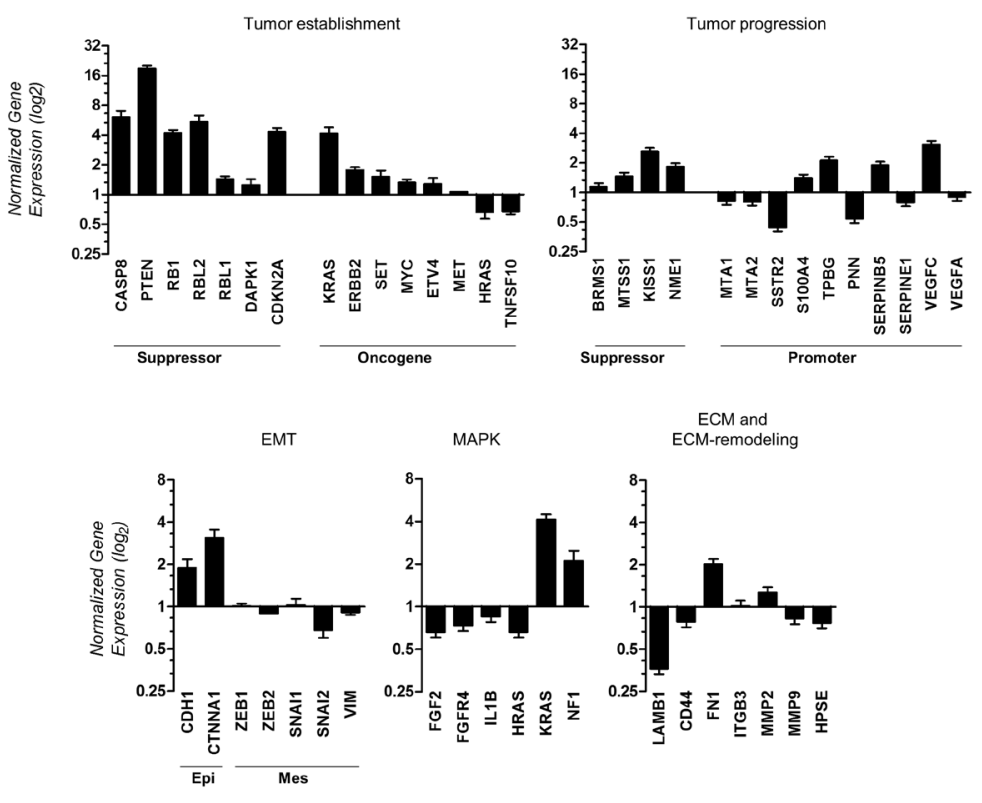

Figure 4: $m i R-654-3 p$ is downregulated during tumor progression of PTC in vivo and regulates cell proliferation and migration of PTC in vitro. a. Thyroid tissue extracted from 5-,12- and 30-weeks old animals (Tg-Braf and WT) was homogenized and total RNA extracted was used for cDNA synthesis. snRNA U6 and RPL19 were used for normalization of miR-654-3p and Zeb1 Zeb2, Snail and Snai2 respectively. b. N-Thy-ORI and TPC-1 cells were transfected or not with miR-654-3p mimetic and after 24,48 , and $72 \mathrm{~h}$, cells were trypsinized and counted. The data is representative of two independent experiments performed in triplicates. c. TPC-1, BCPAP and KTC-2 cells transfected or not with miR-654-3p mimetic were seeded into the upper compartment of modified Boyden chambers with $8 \mu \mathrm{m}$ pore inserts as described in Methods section. Representative images are shown. d. BCPAP cells were transfected with 30nM miR654-3p mimetic. After $48 \mathrm{~h}$, total RNA was extracted and used for cDNA synthesis. Tumor progression and metastasis genes were profiled using Human Tumor Metastasis qPCR Array (Thermo). GAPDH, HPRT and GUSB genes were used as endogenous control. Values in $\mathrm{Y}$ axis reflect the $\log _{2}$ fold change expression levels between BCPAP cells transfected miR-654-3p mimetic with those not transfected. (EMT) Epithelial-to-mesenchymal transition; (Epi) Epithelial markers; (Mes) Mesenchymal markers; (ECM) Extra-cellular Matrix. (A-D) (Tg-Braf) Tg-Braf animals; (WT) wild-type animals; (Mock) cells exposed to the transfection reagent only. Bars represent s.d. * $\mathrm{P}<0.05$; $* * \mathrm{P}<0.01 ; * * * \mathrm{P}<0,001$. 
downregulation of miRNAs from the $14 \mathrm{q} 32$ region concomitant to loss of differentiation and EMT. PTCs generated in Tg-Braf animals recapitulate the intratumor heterogeneity observed in a fraction of PTC patients, including loss of differentiation, TGF $\beta$ driven EMT, tumor-associated macrophages and local invasiveness [17]. Riesco-Eizaguirre and colleagues have shown that BRAFT1799A mutation induces repression of thyroid-differentiation gene NIS through the activation of a TGF- $\beta$ autocrine loop, leading to radioiodine resistance and poor prognosis [21]. We have shown that miRNAs from $14 \mathrm{q} 32$ region target EMT-related genes, which could contribute with the aggressive phenotype observed in these tumors.

Although the deregulation of select individual miRNAs from the $14 \mathrm{q} 32$ region has been shown in PTC cell lines [22, 23], large scale analysis of miRNA expression in vivo combined with data mining of the TCGA project allowed us to identify the synchronized downregulation of miRNAs limited to the $14 \mathrm{q} 32$ region spanning from the $D L K 1$ to $D I O 3$ genes, also known as the $D L K 1-D I O 3$ region. The coordinated downregulation of miRNAs from the $14 \mathrm{q} 32$ region in PTC is aligned with the observation of a large polycistronic transcript comprising the largest miRNA cluster in the human genome [24, 25].

Several studies have shown downregulation of miRNAs from the $14 \mathrm{q} 32$ region in different types of cancer, such as ovarian, breast and prostate cancer, and gastrointestinal stromal tumor, [26-29], with significant correlations to poor prognosis and aggressiveness. Importantly, a tumor suppressor role has been recognized in several of the downregulated miRNAs from the $14 \mathrm{q} 32$ region through the targeting of key oncogenes in glioblastoma, neuroblastoma, metastatic lung cancer, hepatic cancer, pituitary adenoma and rhabdomyosarcoma [30-34]. For example, restoration of miR-370 expression led to downregulation of FOXM1 in acute myeloid leukemia, promoting cell growth arrest and senescence [35]. In contrast, miRNAs from the $14 \mathrm{q} 32$ region may act as oncogenes as well [3638], suggesting that these miRNAs may have different biological roles depending on the tissue of origin and genetic background.

The mechanisms underlying modulation of this region remain to be fully understood. In this study, increased expression of $m i R-203$ observed in thyroid cancer cell lines excludes the existence of terminal deletion of chromosome 14. TCGA data shows homodeletion of the $14 \mathrm{q} 32$ region in one patient sample, however without heterozygosis status. Additionally, despite a large number of oncogenic mutations that have been recently identified in poorly differentiated (PDTC) and anaplastic thyroid carcinomas (ATC), no genetic alteration spanning the $14 \mathrm{q} 32$ region was observed [39].
The relationship between MAPK pathway and the 14q32 region remains uncertain. Cahill and colleagues have shown that human derived BRAFT1799A- and RET/ $P T C$-bearing thyroid tumor cells, KAT10 and TPC-1 respectively, express lower levels of $14 \mathrm{q} 32$-encoded miRNAs $m i R-323-3 p$, miR-370-5p, miR-127-3p, miR299 and miR-154 [22, 23]. However, the in vitro acute transfection of each oncogene into the normal thyroid cell line N-Thy-ORI did not show the same level of impairment of expression of these miRNAs. This observation, combined with the long-term (12 weeks) downregulation of 14q32-encoded miRNAs during progression of PTC in Tg-Braf mice suggests that other mechanisms than the driver oncogene activation may play an additional role on the silencing of $14 \mathrm{q} 32$ genes. Recent studies have shown that epigenetic changes, such as DNA methylation and chromatin remodeling by IncRNA-mediated mechanisms, may participate in regulating the expression of the $14 \mathrm{q} 32$-encoded miRNAs $[28,40]$. Also, imprinting imbalance could lead to the differential modulation of paternally and maternally expressed genes from the $14 \mathrm{q} 32$ region and could explain, at least in part, the increased levels of $\mathrm{DIO} 3$ observed in some PTC samples [41, 42]. Altogether, these data suggest that multiple mechanisms other than genetic mutations or chromosomal loss might be involved in the regulation of $14 \mathrm{q} 32$-encoded miRNAs in thyroid cancer.

Due to the large number of miRNAs present in the $14 \mathrm{q} 32$ region that are concomitantly deregulated, the miRNA:target interaction pattern may lead to a complex regulatory network that may include thousands of potential targets. Our GSEA (Figure 3) indicates that miRNAs from the $14 \mathrm{q} 32$ region could contribute to tumor progression and metastasis by targeting key regulators of biological processes involved in tumorigenesis and tumor progression, such as cell adhesion and migration, proliferation, hypoxic response and wound healing. miR-654-3p, which has been described as a tumor suppressor miRNA in prostate cancer cell lines [28], is downregulated during PTC progression in the Tg-Braf model and in human PTC samples. Our functional analysis indicates that miR-654$3 p$ markedly decreases cell proliferation and increases apoptosis, possibly by restoring the expression of key tumor suppressor genes. Although no morphological changes were observed, transfection of miR-654-3p increased the expression of $C D H 1$ and $C T N N A 1$, and decreased the expression of $S N A I 2$, which is consistent with an "epithelial" genetic program. Importantly, the levels of key modulators of tumor progression, such as ECM and ECM-remodeling genes, as well as metastasissuppressing and -promoting genes, were restored after transfection of a $m i R-654-3 p$ mimetic. 
In conclusion, we have identified the global downregulation of miRNAs from $14 \mathrm{q} 32$ region in a mouse model of PTC progression and in human PTC samples. The potential network modulated by these miRNAs may contribute to important aspects of cancer development and progression, such as cell adhesion, migration, angiogenesis and proliferation. Finally, in vitro functional analyses highlight tumor suppressor properties for $m i R-654-3 p$ in PTC. Due to the large number of potential key cancerrelated targets, further functional analyses of miRNAs from the $14 \mathrm{q} 32$ region may be of great interest for the development of new therapeutic strategies for thyroid cancer.

\section{MATERIALS AND METHODS}

\section{Cell lines and animals}

The PTC-derived cell line TPC-1, which spontaneously harbors RET/PTC1 rearrangement, was kindly provided by Dr James A Fagin (Human Oncology and Pathogenesis Program, Memorial Sloan-Kettering Cancer Center, New York, NY, USA). BCPAP, derived from BRAFT1799A-positive PTC was kindly donated by Dr Massimo Santoro (Medical School, University 'Federico II' of Naples, Naples, Italy). The ATC-derived cell line KTC-2, also harboring BRAFT1799A oncogene was kindly provided by Dr. Norisato Mitsutake (Nagasaki University Graduate School of Biomedical Sciences, Nagasaki, Japan). The origin and genetic background of these cell lines has been recently authenticated [43, 44]. Immortalized non-tumoral thyroid follicular cells N-ThyORI were purchased from Sigma and maintained in RPMI (Invitrogen) supplemented with 10\% FBS and $2 \mathrm{mM}$ L-glutamine (Invitrogen). TPC-1 and BCPAP cells were cultivated as previously described [45]. KTC-2 cells were maintained in RPMI supplemented with 5\% FBS. All cell lines were cultivated with $100 \mathrm{U} / \mathrm{ml}$ penicillin, $1 \mu \mathrm{g} / \mathrm{ml}$ streptomycin and $100 \mu \mathrm{g} / \mathrm{ml}$ amphotericin at $37^{\circ} \mathrm{C}$ and in a 5\% CO2 atmosphere.

FVB transgenic mice, harboring the thyroid-targeted expression of BRAFT1799A oncogene [16] as well as their wild-type counterparts were donated by Dr James Fagin (Human Oncology and Pathogenesis Program, Memorial Sloan-Kettering Cancer Center, New York, NY, USA), and FVB WT mice were used as control. Animal experimentation was conducted in accordance with guidelines from the Ethical Committee of the Institute of Biomedical Sciences (n\#134/ p93/ b2), University of São Paulo, Brazil.

\section{Gene expression analysis}

For the large scale analysis of miRNA expression, thyroid glands were surgically removed from euthanized animals at different time points (1, 5, 12 and 30 weeks) and embedded in RNA Later (Ambion). Total RNA was phenol-chloroform-extracted using TRIzol reagent (Invitrogen) from homogenized thyroid tissue samples according to the manufacturer's instructions. Small RNA fraction was purified using miRNeasy kit (Qiagen) and integrity was verified using Agilent small RNA chip in Agilent Bioanalyzer (Agilent). MiRNA expression quantification was performed using miScript II RT kit for reverse transcription and murine miScript PCR Arrays (Qiagen) for mature miRNA amplification, according to manufacturer's instructions. Data analysis was performed using the online software PCR Array Data Analysis Web Portal (http://pcrdataanalysis.sabiosciences.com/ mirna/arrayanalysis.php). The melting curve of each amplification product was manually checked to assure the specificity of the reaction. In order to increase reliability, the standard deviation for the $\mathrm{Ct}$ values of the 5 genes provided as endogenous controls were calculated and the endogenous gene with the highest value was discarded.

For quantification of miRNA and mRNA expression in vitro, $1 \times 10^{5}$ cells were seeded into $60 \mathrm{~mm}$ dishes. Forty-eight hours after transfection of miRNA mimetic, total RNA was phenol-chloroform-extracted using TRIzol reagent (Invitrogen) according to the manufacturer's instructions. For miRNA expression analysis $10 \mathrm{ng}$ of total RNA was reverse transcribed using TaqMan ${ }^{\circledR}$ MicroRNA Reverse Transcription kit (Applied Biosystems) according to the manufacturer's instructions. MiRNA expression was detected using TaqMan Universal PCR Master Mix No AmpErase UNG (Applied Biosystems). For the quantification of mRNA expression $2 \mu \mathrm{g}$ of total RNA was reverse transcribed using MMLV Reverse transcription kit (Life). RNA U6 small nuclear 2 (RNU6B) and RPL19 genes were used as endogenous controls for normalization of miRNA and mRNA input, respectively. The expression of metastasis-related genes was quantified using Human Tumor Metastasis Taqman ${ }^{\circledR}$ Array plate (ThermoFischer), according with manufacturer's instructions. All amplification reactions were performed using universal cycling conditions in ViiA-7 Real-Time PCR System (Applied Biosystems). RT primers part numbers for miRNA (Applied Biosystems) and the oligonucleotides used for qPCR reaction are shown in Supplementary Table 1. The differential gene expression was calculated according with Pfafll [46].

\section{Data mining of the Cancer Genome Atlas}

miRNA (miRNASeq) and mRNA (RNASeqV2) Gene expression, Copy Number Variation (CNV Lowpass DNA-Seq) and clinical data were downloaded in "level 3" format from TCGA web portal (https://cancergenome. nih.gov). miRNA and mRNA differential expression was calculated using Normalized Read count data for each individual sample. For miRNA and mRNA expression analysis, transcripts were considered not valid when 42 or 
more $(75 \%)$ pairs, out of 57 PTC versus matched normal tissue, had not valid fold changes. In total, 579 out of 1,046 miRNAs were excluded and 467 were considered valid. Also, mRNA transcripts whose median expression were $<=5$ reads across all 114 samples (57 PTC and paired normal tissue) were excluded $(5,142$ out of 20,502$)$.

\section{Gene Set Enrichment Analysis}

The miRNA-target prediction was performed using miRWalk program (http://www.umm.uni-heidelberg.de/ apps/zmf/mirwalk/), accessing a total of 12 algorithms. To increase stringency, one interaction was considered valid when predicted by TargetScan algorithm and 7 or more algorithms. The list of potential targets was then compared with RNA-seq dataset downloaded from TCGA. Upregulated ( $>1.25$ fold) mRNA targets were selected and submitted to DAVID web tool (https://david.ncifcrf.gov/). Biological processes enriched among the target genes potentially targeted by miRNAs from region $14 q 32$ were identified. The enrichment of common targets between each miRNA from 14q32 region was calculated using Fisher's Exact Test or Fisher's Exact Test of Chi-square, if sample size is too large for the Fisher test.

\section{Transient transfection of miR-654-3p mimetic}

Normal and tumor thyroid cells were transfected with $m i R-654-3 p$ miRVana mimic (Life Technologies) at concentration of $10-30 \mathrm{nM}$. The concentration of $30 \mathrm{nM}$ was proved to be more efficient and used for further experiments. Transfection was performed $24 \mathrm{~h}$ after seeding using Lipofectamine 2000® (Invitrogen). Cells exposed to transfection reagent only were used as control and denominated Mock.

\section{Cell proliferation, migration and apoptosis}

Cell counting, migration and apoptosis analyses were performed as previously described, with modifications [45, 47]. Growth curve and apoptosis were measured using Guava EasyCyte Mini Flow Cytometer (Guava Technologies). Migrating cells were visualized after staining with $0.5 \%$ Crystal Violet in Ethanol in Nikon Eclipse E600 microscope.

\section{Statistical analysis}

Statistical analysis was performed using GraphPad Prism Software version 5.00 (San Diego, CA, USA). After the test for normality using Kolmogrov-Smirnov test, samples were compared using non-parametric Mann-Whitney test, when they presented non-Gaussian distribution, and using Students $\mathrm{t}$ test, when they p-resented Gaussian distribution. Differences were considered significant when $\mathrm{P}<0.05$.

\section{ACKNOWLEDGMENTS}

The authors thank Dr. James A. Fagin (Memorial Sloan-Kettering Cancer Center, MSKCC, USA) and Dr. Jeff A. Knauf (MSKCC) for the donation of TgBraf animals and TPC cell line, Dr. Massimo Santoro (University "Federico II" of Naples, Italy) for donation of BCPAP cell line and Dr. Norisato Mitsutake (Nagasaki University, Japan) for donation of KTC-2 cell line. The authors also thank Dr. Cesar S. Fuziwara (University of Sao Paulo, USP, Brazil) for the help with thyroidectomy and Marley J. Silva (USP) for genotyping procedures in Tg-Braf animals.

\section{CONFLICTS OF INTEREST}

The authors declare no conflicts of interest that could prejudice the impartiality of this work.

\section{FUNDING}

This study was funded by the São Paulo Research Foundation (http://fapesp.br/): FAPESP grants 11/52051-2 (MVG) and 2011/50732-2 (ETK); and National Council for Scientific and Technological Development (http://cnpq. br/): CNPq grants 308527/2013-5; 458505/2014-5 (ETK).

\section{Author contribution}

Study and Experiment design: MVG, ETK; Experimentation: MVG; Data analysis; MVG, ETK, HIN; Elaboration of manuscript: MVG, ETK.

\section{REFERENCES}

1. Siegel RL, Miller KD, Jemal A. Cancer statistics, 2016. CA Cancer J Clin. 2016; 66:7-30.

2. Fröhlich E, Wahl R. The current role of targeted therapies to induce radioiodine uptake in thyroid cancer. Cancer Treat Rev. 2014; 40:665-674.

3. Fugazzola L, Puxeddu E, Avenia N, Romei C, Cirello V, Cavaliere A, Faviana P, Mannavola D, Moretti S, Rossi S, Sculli M, Bottici V, Beck-Peccoz P, et al. Correlation between B-RAFV600E mutation and clinico-pathologic parameters in papillary thyroid carcinoma. data from a multicentric Italian study and review of the literature. Endocr Relat Cancer. 2006; 13:455-464.

4. Nikiforova MN, Kimura ET, Gandhi M, Biddinger PW, Knauf JA, Basolo F, Zhu Z, Giannini R, Salvatore G, Fusco A, Santoro M, Fagin JA, Nikiforov YE. BRAF mutations in thyroid tumors are restricted to papillary carcinomas and anaplastic or poorly differentiated carcinomas arising from papillary carcinomas. J Clin Endocrinol Metab. 2003; 88:5399-5404. 
5. Riesco-Eizaguirre G, Gutiérrez-Martínez P, GarcíaCabezas MA, Nistal M, Santisteban P. The oncogene BRAF $\mathrm{V} 600 \mathrm{E}$ is associated with a high risk of recurrence and less differentiated papillary thyroid carcinoma due to the impairment of $\mathrm{Na}+/ \mathrm{I}$ - targeting to the membrane. Endocr Relat Cancer. 2006; 13:257-269.

6. Bartel DP. MicroRNAs. genomics, biogenesis, mechanism, and function. Cell. 2004; 116:281-297.

7. He H, Jazdzewski K, Li W, Liyanarachchi S, Nagy R, Volinia S, Calin GA, Liu CG, Franssila K, Suster S, Kloos RT, Croce CM, de la Chapelle A. The role of microRNA genes in papillary thyroid carcinoma. Proc Natl Acad Sci U S A. 2005; 102:19075-19080.

8. Pallante P, Visone R, Ferracin M, Ferraro A, Berlingieri MT, Troncone G, Chiappetta G, Liu CG, Santoro M, Negrini M, Croce CM, Fusco A. MicroRNA deregulation in human thyroid papillary carcinomas. Endocr Relat Cancer. 2006; 13:497-508.

9. Visone R, Pallante P, Vecchione A, Cirombella R, Ferracin M, Ferraro A, Volinia S, Coluzzi S, Leone V, Borbone E, Liu CG, Petrocca F, Troncone G, et al. Specific microRNAs are downregulated in human thyroid anaplastic carcinomas. Oncogene. 2007; 26:7590-7595.

10. Nikiforova MN, Tseng GC, Steward D, Diorio D, Nikiforov YE. MicroRNA expression profiling of thyroid tumors. biological significance and diagnostic utility. J Clin Endocrinol Metab. 2008; 93:1600-1608.

11. Lee JC, Zhao JT, Clifton-Bligh RJ, Gill A, Gundara JS, Ip JC, Glover A, Sywak MS, Delbridge LW, Robinson BG, Sidhu SB. MicroRNA-222 and microRNA-146b are tissue and circulating biomarkers of recurrent papillary thyroid cancer. Cancer. 2013; 119:4358-4365.

12. Benetatos L, Voulgaris E, Vartholomatos G. DLK1-MEG3 imprinted domain microRNAs in cancer biology. Crit Rev Eukaryot Gene Expr. 2012; 22:1-15.

13. Benetatos L, Hatzimichael E, Londin E, Vartholomatos G, Loher P, Rigoutsos I, Briasoulis E. The microRNAs within the DLK1-DIO3 genomic region. involvement in disease pathogenesis. Cell Mol Life Sci. 2013; 70:795-814.

14. Ioannides Y, Lokulo-Sodipe K, Mackay DJ, Davies JH, Temple IK. Temple syndrome. improving the recognition of an underdiagnosed chromosome 14 imprinting disorder. an analysis of 51 published cases. J Med Genet. 2014; 51:495-501.

15. Severi G, Bernardini L, Briuglia S, Bigoni S, Buldrini B, Magini P, Dentici ML, Cordelli DM, Arrigo T, Franzoni E, Fini S, Italyankina E, Loddo I, et al. New patients with temple syndrome caused by $14 q 32$ deletion. Genotypephenotype correlations and risk of thyroid cancer. Am J Med Genet A. 2015; 170A:162-9.

16. Knauf JA, Ma X, Smith EP, Zhang L, Mitsutake N, Liao XH, Refetoff S, Nikiforov YE, Fagin JA. Targeted expression of BRAFV600E in thyroid cells of transgenic mice results in papillary thyroid cancers that undergo dedifferentiation. Cancer Res. 2005; 65:4238-4245.

17. Knauf JA, Sartor MA, Medvedovic M, Lundsmith E, Ryder M, Salzano M, Nikiforov YE, Giordano TJ, Ghossein RA, Fagin JA. Progression of BRAF-induced thyroid cancer is associated with epithelial-mesenchymal transition requiring concomitant MAP kinase and TGF $\beta$ signaling. Oncogene. 2011; 30:3153-3162.

18. Zhang J, Liu Y, Liu Z, Wang XM, Yin DT, Zheng LL, Zhang DY, Lu XB. Differential expression profiling and functional analysis of microRNAs through stage I-III papillary thyroid carcinoma. Int J Med Sci. 2013; 10:585-592.

19. Yang Z, Yuan Z, Fan Y, Deng X, Zheng Q. Integrated analyses of microRNA and mRNA expression profiles in aggressive papillary thyroid carcinoma. Mol Med Rep. 2013; 8:1353-1358.

20. Geraldo MV, Kimura ET. Integrated Analysis of Thyroid Cancer Public Datasets Reveals Role of Post-Transcriptional Regulation on Tumor Progression by Targeting of Immune System Mediators. PLoS One. 2015; 10:e0141726.

21. Riesco-Eizaguirre G, Rodríguez I, De la Vieja A, Costamagna E, Carrasco N, Nistal M, Santisteban P. The BRAFV600E oncogene induces transforming growth factor beta secretion leading to sodium iodide symporter repression and increased malignancy in thyroid cancer. Cancer Res. 2009; 69:8317-8325.

22. Cahill S, Smyth P, Finn SP, Denning K, Flavin R, O'Regan EM, Li J, Potratz A, Guenther SM, Henfrey R, O'Leary JJ, Sheils O. Effect of ret/PTC 1 rearrangement on transcription and post-transcriptional regulation in a papillary thyroid carcinoma model. Mol Cancer. 2006; 5:70.

23. Cahill S, Smyth P, Denning K, Flavin R, Li J, Potratz A, Guenther SM, Henfrey R, O'Leary JJ, Sheils O. Effect of BRAFV600E mutation on transcription and posttranscriptional regulation in a papillary thyroid carcinoma model. Mol Cancer. 2007; 6:21.

24. Glazov EA, McWilliam S, Barris WC, Dalrymple BP. Origin, evolution, and biological role of miRNA cluster in DLK-DIO3 genomic region in placental mammals. Mol Biol Evol. 2008; 25:939-948.

25. Fiore R, Khudayberdiev S, Christensen M, Siegel G, Flavell SW, Kim TK, Greenberg ME, Schratt G. Mef2-mediated transcription of the miR379-410 cluster regulates activitydependent dendritogenesis by fine-tuning Pumilio2 protein levels. EMBO J. 2009; 28:697-710.

26. Kelly L, Bryan K, Kim SY, Janeway KA, Killian JK, Schildhaus HU, Miettinen M, Helman L, Meltzer PS, van de Rijn M, Debiec-Rychter M, O'Sullivan M. NIH Pediatric and Wild-Type GIST Clinic. Post-transcriptional dysregulation by miRNAs is implicated in the pathogenesis of gastrointestinal stromal tumor [GIST]. PLoS One. 2013; 8:e64102. 
27. Anaya-Ruiz M, Bandala C, Perez-Santos JL. miR-485 acts as a tumor suppressor by inhibiting cell growth and migration in breast carcinoma T47D cells. Asian Pac J Cancer Prev. 2013; 14:3757-3760.

28. Formosa A, Markert EK, Lena AM, Italiano D, FinazziAgro' E, Levine AJ, Bernardini S, Garabadgiu AV, Melino G, Candi E. MicroRNAs, miR-154, miR-299-5p, miR-376a, miR-376c, miR-377, miR-381, miR-487b, miR-485-3p, miR-495 and miR-654-3p, mapped to the 14q32.31 locus, regulate proliferation, apoptosis, migration and invasion in metastatic prostate cancer cells. Oncogene. 2014; 33:5173-5182.

29. Zehavi L, Avraham R, Barzilai A, Bar-Ilan D, Navon R, Sidi Y, Avni D, Leibowitz-Amit R. Silencing of a large microRNA cluster on human chromosome $14 \mathrm{q} 32$ in melanoma. biological effects of mir-376a and mir-376c on insulin growth factor 1 receptor. Mol Cancer. 2012; 11:44.

30. Niu CS, Yang Y, Cheng CD. MiR-134 regulates the proliferation and invasion of glioblastoma cells by reducing Nanog expression. Int J Oncol. 2013; 42:1533-1540.

31. Chen L, Zhang J, Feng Y, Li R, Sun X, Du W, Piao X, Wang H, Yang D, Sun Y, Li X, Jiang T, Kang C, et al. MiR-410 regulates $\mathrm{MET}$ to influence the proliferation and invasion of glioma. Int J Biochem Cell Biol. 2012; 44:1711-1717.

32. Lim L, Balakrishnan A, Huskey N, Jones KD, Jodari M, Ng R, Song G, Riordan J, Anderton B, Cheung ST, Willenbring H, Dupuy A, Chen X, et al. MicroRNA-494 within an oncogenic microRNA megacluster regulates G1/S transition in liver tumorigenesis through suppression of mutated in colorectal cancer. Hepatology. 2014; 59:202-215.

33. Gu Y, Cheng Y, Song Y, Zhang Z, Deng M, Wang C, Zheng G, He Z. MicroRNA-493 suppresses tumor growth, invasion and metastasis of lung cancer by regulating E2F1. PLoS One. 2014; 9:e102602.

34. Sun M, Huang F, Yu D, Zhang Y, Xu H, Zhang L, Li L, Dong L, Guo L, Wang S. Autoregulatory loop between TGF- $\beta 1 / \mathrm{miR}-411-5 \mathrm{p} / \mathrm{SPRY} 4$ and MAPK pathway in rhabdomyosarcoma modulates proliferation and differentiation. Cell Death Dis. 2015; 6:e1859.

35. Zhang X, Zeng J, Zhou M, Li B, Zhang Y, Huang T, Wang $\mathrm{L}$, Jia J, Chen C. The tumor suppressive role of miRNA370 by targeting FoxM1 in acute myeloid leukemia. Mol Cancer. 2012; 11:56.

36. Chien WW, Domenech C, Catallo R, Kaddar T, Magaud JP, Salles G, Ffrench M. Cyclin-dependent kinase 1 expression is inhibited by p16(INK4a) at the post-transcriptional level through the microRNA pathway. Oncogene. 2011; 30:1880-1891.

37. Dixon-McIver A, East P, Mein CA, Cazier JB, Molloy G, Chaplin T, Andrew Lister T, Young BD, Debernardi S.
Distinctive patterns of microRNA expression associated with karyotype in acute myeloid leukaemia. PLoS One. 2008; 3:e2141.

38. Chabre O, Libé R, Assie G, Barreau O, Bertherat J, Bertagna X, Feige JJ, Cherradi N. Serum miR-483-5p and miR-195 are predictive of recurrence risk in adrenocortical cancer patients. Endocr Relat Cancer. 2013; 20:579-594.

39. Landa I, Ibrahimpasic T, Boucai L, Sinha R, Knauf JA, Shah RH, Dogan S, Ricarte-Filho JC, Krishnamoorthy GP, Xu B, Schultz N, Berger MF, Sander C, et al. Genomic and transcriptomic hallmarks of poorly differentiated and anaplastic thyroid cancers. J Clin Invest. 2016; 126:1052-1066.

40. Li L, Liu B, Wapinski OL, Tsai MC, Qu K, Zhang J, Carlson JC, Lin M, Fang F, Gupta RA, Helms JA, Chang HY. Targeted disruption of Hotair leads to homeotic transformation and gene derepression. Cell Rep. 2013; 5:3-12.

41. Romitti M, Wajner SM, Ceolin L, Ferreira CV, Ribeiro RV, Rohenkohl HC, Weber SdeS, Lopez PL, Fuziwara CS, Kimura ET, Maia AL. MAPK and SHH pathways modulate type 3 deiodinase expression in papillary thyroid carcinoma. Endocr Relat Cancer. 2016; 23:135-146.

42. Romitti M, Wajner SM, Zennig N, Goemann IM, Bueno AL, Meyer EL, Maia AL. Increased type 3 deiodinase expression in papillary thyroid carcinoma. Thyroid. 2012; 22:897-904.

43. Schweppe RE, Klopper JP, Korch C, Pugazhenthi U, Benezra M, Knauf JA, Fagin JA, Marlow LA, Copland JA, Smallridge RC, Haugen BR. Deoxyribonucleic acid profiling analysis of 40 human thyroid cancer cell lines reveals cross-contamination resulting in cell line redundancy and misidentification. J Clin Endocrinol Metab. 2008; 93:4331-4341.

44. Saiselet M, Floor S, Tarabichi M, Dom G, Hébrant A, van Staveren WC, Maenhaut C. Thyroid cancer cell lines. an overview. Front Endocrinol (Lausanne). 2012; 3:133.

45. Geraldo MV, Yamashita AS, Kimura ET. MicroRNA miR146b-5p regulates signal transduction of TGF-beta by repressing SMAD4 in thyroid cancer. Oncogene. 2012; 31:910-1922.

46. Pfaffl MW. A new mathematical model for relative quantification in real-time RT-PCR. Nucleic Acids Res. 2001; 29:e45.

47. Lima CR, Geraldo MV, Fuziwara CS, Kimura ET, Santos MF. MiRNA-146b-5p upregulates migration and invasion of different Papillary Thyroid Carcinoma cells. BMC Cancer. 2016; 16:108. 